\title{
Caustic design in periodic lattices
}

\author{
Nikolaos K. Efremidis* and Ioannis D. Chremmos \\ Department of Applied Mathematics, University of Crete, 71409 Heraklion, Crete, Greece \\ ${ }^{*}$ Corresponding author: nefrem@tem.uoc.gr
}

Received January 11, 2012; accepted February 24, 2012;

posted February 29, 2012 (Doc. ID 161376); published March 30, 2012

\begin{abstract}
We study curved trajectory dynamics and design in discrete array settings. We find that beams with power law phases produce curved caustics associated with the fold and cusp type catastrophes. A parabolic phase produces a focus that suffers from spherical aberrations. More important, we find that by designing the initial phase or wavefront of the beam we can construct trajectories with pure power law caustics as well as aberration-free focusing of discrete waves. (c) 2012 Optical Society of America
\end{abstract}

OCIS codes: $130.2790,230.7380,350.5500$.

Light propagation along curved trajectories is a topic of increased interest over the past few years. A main example is the Airy wave that follows a parabolic trajectory and is the only one-dimensional diffraction-free solution of the Schrödinger equation [1]. Airy beams were introduced in optics in [2,3], where it was shown that exponentially apodized Airy waves can easily be generated in the Fourier space by applying a cubic phase to a Gaussian beam. Along with apodization, their power becomes finite, and thus they lose their nondiffracting character. However, apodized Airy waves can propagate for several diffraction lengths before they become significantly distorted. A different class of diffraction-free accelerating waves are the two-dimensional (2D) parabolic beams as predicted in [4]. Curved light waves have found potential applications in particle manipulation [5], filament generation [6], plasmonics [7,8] and near field imaging [9], and optical bullet formation [10,11]. In cylindrical coordinates the on-axis interaction of radial Airy-type caustics leads to the phenomenon of abrupt autofocusing, i.e., to the sudden increase of the intensity of light by several orders of magnitude at the focal point [12-15].

Light beams can follow different families of curved trajectories, including the general power law $[13,16]$. The cost of this adjustability is the loss of the diffraction resisting character of the Airy wave. Such light trajectories can be analyzed by utilizing caustics and catastrophe theory $[17,18]$. In periodic index configurations, such as waveguide arrays, the behavior of light waves becomes effectively "discretized." For a comprehensive review on linear and nonlinear dynamics in periodic lattices see [19] and references therein. In [20] a review on the modulated lattice is also presented. A discrete system with a linear index gradient is known to support Wannier-Stark states [21]. These states, in the absence of the linear potential, have been shown to propagate in waveguide arrays along curved trajectories [22].

In this letter, we explore the possibilities of generating caustics in discrete waveguide settings. In the case where the initial phase of the beam is power law, the resulting curved trajectory is parametrically determined. According to the value of the exponent of the phase, the associated catastrophe can be a fold or a cusp. In addition, in the case of a parabolic wavefront, the resulting focusing effect suffers from spherical aberrations. More important, we find that the discrete beam trajectory can be engineered by appropriately selecting the initial phase.
Thus, we can generate pure power law caustics as well as aberration-free discrete focusing of light.

Let us consider the coupled mode theory equations

$$
i \dot{u}_{n}+\kappa\left(u_{n-1}+u_{n+1}\right)=0
$$

that describe the propagation in a one-dimensional periodic lattice. In this potential-free discretized version of the Schrödinger equation, $u_{n}$ describes the field amplitude in waveguide $n, \dot{u}_{n}=d u_{n} / d z, z$ is the propagation direction, and $\kappa$ is the coupling coefficient between adjacent waveguides. Equation (1) supports plane wave solutions $e^{i(q n-k(q) z)}$ with a dispersion relation $k(q)=-2 \kappa \cos q$. Using the Fourier transform pair we find that

$$
u_{n}(z)=\frac{1}{2 \pi} \sum_{m=-\infty}^{\infty} u_{m}(0) \int_{0}^{2 \pi} e^{i q(n-m)+2 i \kappa \cos (q) z} \mathrm{~d} q
$$

We define a continuous (real) variable $\xi$ such that $u(\xi, 0)=u_{n}(0)$ if $\xi$ is an integer and assume that $u(\xi, 0)$ is slowly varying with $\xi$ and goes to zero as $\xi \rightarrow \pm \infty$. Physically, $\xi$ represents the transverse coordinate at the input plane. Under these assumptions, and using an amplitude and phase decomposition $u(\xi, 0)=$ $A(\xi) \exp [i \phi(\xi)]$, the Euler-type sum in Eq. (2) can be approximated by the following integral:

$$
u(x, z)=\frac{1}{2 \pi} \int_{-\infty}^{\infty} \int_{0}^{2 \pi} A(\xi) e^{i \Psi} \mathrm{d} q \mathrm{~d} \xi,
$$

where $\Psi=\phi(\xi)+q(x-\xi)+2 \kappa \cos (q) z$. Note that the initial phase distribution $\phi(\xi)$ can be generated by using, for example, a spatial light modulator.

We now apply a stationary phase method in Eq. (3) on the integration variables $q, \xi$, leading to the ray equation

$$
x=\xi+2 \kappa \sin (q(\xi)) z,
$$

where $q(\xi)=\phi^{\prime}(\xi)$ is the local value of the Bloch momentum. Along the caustic trajectory, the phase should be stationary to higher than first order to variations of the initial wavefront. Requiring second order stationarity $\Psi_{\xi \xi} \Psi_{q q}-\Psi_{q \xi}^{2}=0$, we obtain 


$$
2 \approx \kappa \cos (q(\xi)) q^{\prime}(\xi)+1=0
$$

In the continuous limit (Schrödinger or paraxial equation), optical waves with power law phases produce beams with power law trajectories (having, in general, different exponents). Our analysis shows that owing to discreteness this behavior is significantly altered. In particular, we assume that the phase is nonzero for $\xi<0$, i.e.,

$$
\phi(\xi)=-\alpha(-\xi)^{\beta} H(-\xi) / \beta,
$$

where $\beta$ is the chirp parameter, $\alpha$ characterizes the spatial extent of the phase, and $H(x)$ is the Heaviside theta function, leading to $q(\xi)=\alpha(-\xi)^{\beta-1} H(-\xi)$. Note that the phase can be either exponential $e^{i \phi}$ or sinusoidal $\sin \phi=\left(e^{i \phi}-e^{-i \phi}\right) /(2 i)$. In the latter case the additional $e^{-i \phi}$ term gives rise to rays propagating in the opposite direction that are not associated with caustics. Using Eqs. (4) and (5), we find that the trajectory of the caustic is parametrically determined as a function of $\xi$ as

$$
\begin{gathered}
z=\frac{(-\xi)^{2-\beta}}{2 \alpha(\beta-1) \kappa \cos \left(\alpha(-\xi)^{\beta-1}\right)}, \\
x=\xi+\frac{\tan \left(\alpha(-\xi)^{\beta-1}\right)}{\alpha(\beta-1)(-\xi)^{\beta-2}} .
\end{gathered}
$$

We restrict ourselves to rays propagating to the right $\kappa \sin (q(\xi))>0$ that are able to form caustics $\kappa \cos (q(\xi)) q^{\prime}(\xi)<0$. The resulting range of values, assuming that $q(\xi)$ is a decreasing function of $\xi$ with $q(0)=0, \quad$ is $0<q(\xi)<\pi / 2$. If $\beta>1$ [and thus $q(0)=0]$, then only waveguides in the range $\left[\xi_{0}, 0\right]$ can contribute to the caustic, where $\xi_{0}=-[\pi /(2 \alpha)]^{1 /(\beta-1)}$. Alternatively, we can determine $\alpha$ as a function of $\xi_{0}$ via $\alpha=\pi /\left[2\left(-\xi_{0}\right)^{\beta-1}\right]$. For small values of $\xi$ (and thus small values of $x$ ) the caustic trajectory takes the form

$$
x=[(2-\beta) /(\beta-1)][2 \alpha(\beta-1) \kappa z]^{1 /(2-\beta)} .
$$

As expected, Eq. (9) is identical to the caustic trajectory of the paraxial equation with a wavefront given by Eq. (6). On the other hand, as $\xi \rightarrow \xi_{0}$ the caustic asymp-

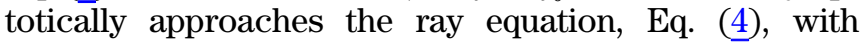
$\xi=\xi_{0}$, i.e.,

$$
x=\xi_{0}+2 \kappa z .
$$

In Fig. 1 typical examples of discrete caustic dynamics generated by power law wavefronts are depicted. In the simulations presented below we set $\kappa=1$. In Figs. 1(a) and $\underline{1(\mathrm{~d})}$, the phase is sinusoidal with exponent $\beta=\overline{3 / 2}$. The parabolic trajectory, and thus the constant acceleration $x^{\prime \prime}(z)$, in the early stage dynamics [Eq. (9)] is characteristic to the Airy wave solution of the paraxial equation. As $z$ increases, the acceleration of the trajectory gradually decreases, and eventually, for large $z$, it goes to zero as the caustic approaches the asymptotic given by Eq. (10). Discrete waves with phase exponents
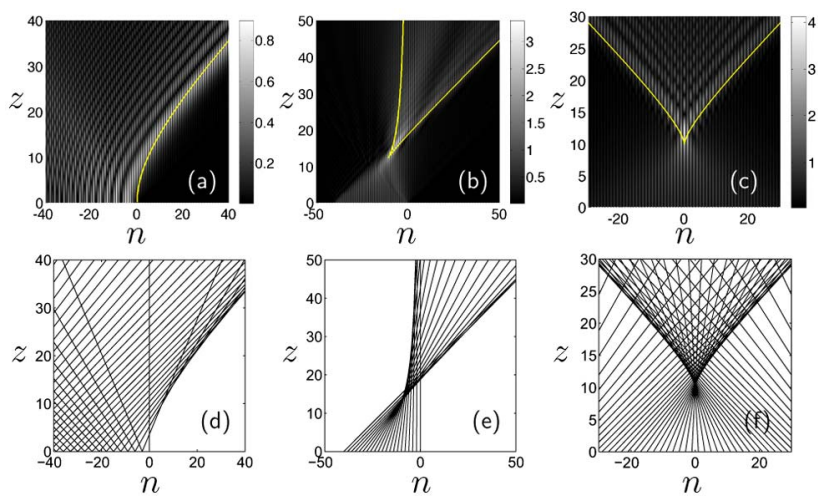

Fig. 1. (Color online) Wave dynamics in the case of a wavefront given by Eq. (6). In (a)-(c) the amplitude profile is shown along with the caustic trajectory, while in (d)-(f) the corresponding ray picture is depicted. In particular, in (a) and (d) $\beta=3 / 2, \xi_{0}=40, A(\xi)=e^{0.02 \xi} H(-\xi)$; in (b) and (e) $\beta=3$, $\xi_{0}=40, A(\xi)=H\left(\xi+\xi_{0}\right) H(-\xi)$; in (c) and (f) $\beta=2, \xi_{0}=20$, $A(\xi)=e^{-\left(\xi / \xi_{0}\right)^{2}}$.

$1<\beta<2$ exhibit qualitatively similar behavior. In terms of catastrophe theory, such a caustic is labeled as a "fold." When $\beta>2$, the behavior is significantly altered since the respective catastrophe becomes a "cusp." A typical example is depicted in Figs. 1(b) and 1(e) for $\beta=3$ and an exponential phase. We see that two caustics are formed that originate from a cusp. The location of the cusp is determined by noting that $z(\xi)$ has a minimum at the cusp $\left[z^{\prime}\left(\xi_{c}\right)=0\right.$ or $\left.\sin (q) q^{2}=\cos (q) q^{\prime \prime}\right]$ leading to $\xi_{c} \approx 25.8$ and $z\left(\xi_{c}\right) \approx 12.4$. Equivalently, we can say that $\xi_{c}$ is a third order stationary phase point of Eq. (3). As a result, the rays emerging from waveguides $-25 \leq n \leq 0$ form the caustic on the left, while the remaining rays $(-40 \leq n \leq-26)$ generate the caustic on the right. In Figs. 1(c) and 1(f) the intermediate case $\beta=2$ with exponential phase $\phi=-\alpha \xi^{2} / 2$ is depicted. In bulk media, such a parabolic phase results in the focusing of the initial wavefront at the focal point. However, because of spherical aberrations caustics might be formed before (positive aberration) or after (negative aberration) the focal point. The situation here is similar to the case of negative spherical aberration: Caustics are formed after the focus, which is given by $z_{f}=1 /[2 \alpha(\beta-1) \kappa]$.

We would like to point out that different types of caustic trajectories can be generated by appropriately engineering the initial phase. Here we are going to consider the following power law caustic trajectories:

$$
x=f(z)=\gamma z^{\delta} .
$$

Using $\xi=f(z)-z f^{\prime}(z)$, which relates the caustic propagation distance $z$ to $\xi$, we can derive the optical wavefront $\phi=\int_{0}^{\xi} q(\xi) \mathrm{d} \xi$, where

$$
q(\xi)=\Re\left\{\arcsin \left[\frac{\gamma^{1 / \delta} \delta}{2 \kappa}\left(\frac{\xi}{1-\delta}\right)^{(\delta-1) / \delta}\right]\right\}
$$

and $\Re$ denotes the real part, reminding us that $\Re \arcsin c=(\pi / 2) \operatorname{sgn}(c)$ if $|c|>1$, where $\operatorname{sgn}(x)$ is the sign function. The optical wavefront that generates the caustic extends from $\xi_{0}$ to 0 where $q\left(\xi_{0}\right)=\pi / 2$. Using 

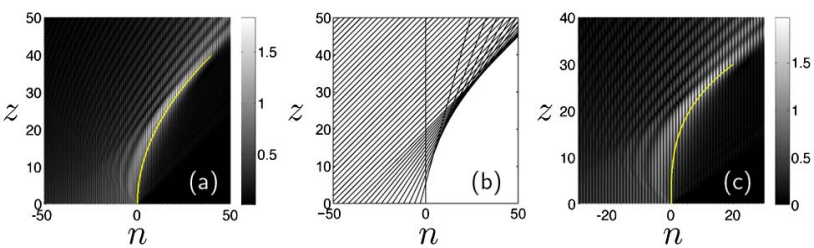

Fig. 2. (Color online) Dynamics of waves that follow power law caustics [Eq. (11)] with $A(\xi)=e^{0.02 \xi} H(-\xi)$. (a) Amplitude profile and the quadratic caustic trajectory, and (b) the corresponding ray picture for $\delta=2, \xi_{0}=40$; (c) amplitude profile and cubic caustic trajectory for $\delta=3, \xi_{0}=40$.
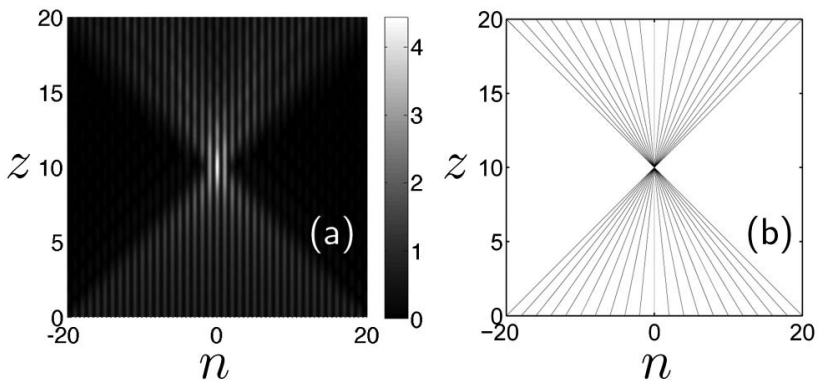

Fig. 3. Discrete focusing of an optical wavefront given by Eq. (13) with $z_{f}=10$ (a) amplitude profile and (b) the corresponding ray picture.

this latter equation we find $\gamma=(2 \kappa / \delta)^{\delta}\left[(1-\delta) / \xi_{0}\right]^{\delta-1}$. Note that the rays can form the predefined caustic, as described by Eq. (11) up to a finite value of the propagation distance given by $z_{0}=z\left(\xi_{0}\right)=-\xi_{0} \delta /[2 \kappa(\delta-1)]$. For $\xi<\xi_{0}$ the rays cannot "bend" any farther [the group velocity $k^{\prime}(q(\xi))$ has a maximum for $\xi=\xi_{0}$ ], and the caustic disappears. In Fig. 2 we see typical examples of power law caustics. In particular, the caustic trajectory in Figs. 2(a) and 2(b) is quadratic while in 2(c) it is cubic. In both cases the caustic extends up to a finite value of the propagation distance.

By engineering the phase of the optical beam, we can eliminate spherical aberrations and produce an ideal focus. In particular, by setting $x=0$ and $z=z_{f}$ (focal point) to the ray equation, we obtain

$$
\phi=\Re\left\{\sqrt{\left(2 \kappa z_{f}\right)^{2}-\xi^{2}}-2 \kappa z_{f}+\xi \arcsin \left(\frac{\xi}{2 \kappa z_{f}}\right)\right\} .
$$

The rays can focus as long as $q(\xi)=\phi_{\xi}(\xi)=$ $-\arcsin \left(\xi /\left(2 \kappa z_{f}\right)\right)$ lies in the range $[-\pi / 2, \pi / 2]$, i.e., for waveguide indices $|n| \leq 2 \kappa z_{f}$. A typical example of discrete focusing is depicted in Fig. 3 .

In conclusion, we have studied beam trajectory dynamics and engineering in discrete optical settings. We have shown that power law phases lead to curved cusp and fold type caustics. A parabolic initial wavefront results in beam focusing with negative spherical aberrations. By engineering the beam wavefront, we found discrete waves with pure power law caustic trajectories as well as aberration-free discrete beam focusing.

This work was supported by the FP7-REGPOT-2009-1 project "Archimedes Center for Modeling, Analysis and Computation" (ACMAC).

\section{References}

1. M. V. Berry and N. L. Balazs, Am. J. Phys. 47, 264 (1979).

2. G. A. Siviloglou and D. N. Christodoulides, Opt. Lett. 32, 979 (2007).

3. G. A. Siviloglou, J. Broky, A. Dogariu, and D. N. Christodoulides, Phys. Rev. Lett. 99, 213901 (2007).

4. M. A. Bandres, Opt. Lett. 33, 1678 (2008).

5. J. Baumgartl, M. Mazilu, and K. Dholakia, Nat. Photon. 2, 675 (2008).

6. P. Polynkin, M. Kolesik, J. V. Moloney, G. A. Siviloglou, and D. N. Christodoulides, Science 324, 229 (2009).

7. A. Salandrino and D. N. Christodoulides, Opt. Lett. 35, 2082 (2010).

8. P. Zhang, S. Wang, Y. Liu, X. Yin, C. Lu, Z. Chen, and X. Zhang, Opt. Lett. 36, 3191 (2011).

9. A. Minovich, A. E. Klein, N. Janunts, T. Pertsch, D. N. Neshev, and Y. S. Kivshar, Phys. Rev. Lett. 107, 116802 (2011).

10. A. Chong, W. H. Renninger, D. N. Christodoulides, and F. W. Wise, Nat. Photon. 4, 103 (2010).

11. D. Abdollahpour, S. Suntsov, D. G. Papazoglou, and S. Tzortzakis, Phys. Rev. Lett. 105, 253901 (2010).

12. N. K. Efremidis and D. N. Christodoulides, Opt. Lett. 35, 4045 (2010).

13. I. Chremmos, N. K. Efremidis, and D. N. Christodoulides, Opt. Lett. 36, 1890 (2011).

14. D. G. Papazoglou, N. K. Efremidis, D. N. Christodoulides, and S. Tzortzakis, Opt. Lett. 36, 1842 (2011).

15. P. Zhang, J. Prakash, Z. Zhang, M. S. Mills, N. K. Efremidis, D. N. Christodoulides, and Z. Chen, Opt. Lett. 36, 2883 (2011).

16. E. Greenfield, M. Segev, W. Walasik, and O. Raz, Phys. Rev. Lett. 106, 213902 (2011).

17. M. V. Berry and C. Upstill, in Progress in Optics, E. Wolf, ed. (North-Holland, 1980), Vol. XVIII, pp. 259-323.

18. Y. A. Kravtsov and Y. I. Orlov, Caustics, Catastrophes and Wave Fields (Springer, 1999).

19. F. Lederer, G. I. Stegeman, D. N. Christodoulides, G. Assanto, M. Segev, and Y. Silberberg, Phys. Rep. 463, 1 (2008).

20. I. L. Garanovich, S. Longhi, A. A. Sukhorukov, and Y. S. Kivshar, arXiv:1107.2992v1 (2011).

21. U. Peschel, T. Persch, and F. Lederer, Opt. Lett. 23, 1701 (1998).

22. R. El-Ganainy, K. G. Makris, M. A. Miri, D. N. Christodoulides, and Z. Chen, Phys. Rev. A 84, 023842 (2011). 